\title{
Two continuous methods for determining a minimal risk path through a minefield
}

\author{
G. N. Mercer ${ }^{1}$ H. S. Sidhu ${ }^{2}$
}

(Received 25 July 2006; revised 18 July 2007)

\begin{abstract}
Two formulations for determining the minimal risk path of a vehicle through a minefield are considered and compared. Firstly, by following a calculus of variations argument a system of four coupled ODEs are derived. Secondly, the trajectory of the vehicle is approximated by a discrete (but large) system of coupled springs and masses resulting in a large but well behaved system of ODEs to solve. $\mathrm{Nu}-$ merical solutions to these systems are investigated. The strengths and weaknesses of each approach are discussed.
\end{abstract}

\section{Contents}

See http://anziamj.austms.org.au/ojs/index.php/ANZIAMJ/article/view/56 for this article, (c) Austral. Mathematical Soc. 2007. Published July 28, 2007. ISSN 1446-8735 
2 Calculus of variations approach

C295

2.1 Formulation . . . . . . . . . . . . . . C295

2.2 Numerical solution . . . . . . . . . . . . . . . C297

3 Springs and masses approach

C300

3.1 Formulation . . . . . . . . . . . . . . . C300

3.2 Numerical solution . . . . . . . . . . . . . . . C302

4 Conclusion

C303

References

C304

\section{Introduction}

The determination of an optimal path trajectory is a fundamental requirement for land, naval, air and space vehicles in both military and civilian applications [5]. Frequently, this optimal trajectory also has constraints such as fuel usage, travel time and travel distance associated with it. This optimal trajectory arises in a number of settings such as minimising risk when travelling through a minefield, minimising detection from enemy radar or minimising aircraft collision in an air traffic control environment [5, 9]. Each application would seek a different balance between the risk and the constraints.

This article investigates the determination of optimal path routes for a single vehicle traversing a minefield from its base to its mission objective. This could be a land vehicle (or even a foot soldier) traversing a landmine field or a ship traversing a region with sea mines. We make some assumptions about the mines and the vehicle dynamics to make the problem amenable to a general solution technique. The locations of the mines are considered to be known. The risk from each mine is assumed to be proportional to the reciprocal of the square of distance from the mine to the current position along 
the trajectory. The vehicle is assumed to have the same risk in all directions so that the shape of the vehicle is not important. The mines and vehicle are assumed to be in the same horizontal plane (sea surface, land surface) so that the problem is two dimensional. This latter criteria is easily relaxed for scenarios such as submerged mines or submarines by including a vertical component in the risk formula. For simplicity it is assumed that the vehicle moves at a constant speed. Any time or fuel constraints can then be expressed as a constraint on the length of the optimal trajectory. It is assumed that the vehicle can respond to any direction change instantaneously.

We study this problem in a continuous variable setting so we allow the course of the vehicle to be continuously changing in order to minimise the risk. This is in contrast to previous work in this area $[2,3,5,8,10]$, who have all treated the trajectory as a series of discrete steps and used discrete optimisation techniques on this approximate problem. A serious drawback of such a discretisation approach is the determination of suboptimal paths due to the gridding scheme incorporated in such methods [7]. One way to improve this is to increase the number of discretisation points, but this is often not feasible (particularly for on-board systems) due to high computational costs.

\section{Calculus of variations approach}

\section{$2.1 \quad$ Formulation}

Consider the case of $N$ radars at positions $(x, y)=\left(a_{i}, b_{i}\right)$ for $i=1, \ldots, N$. The vehicle starts at point $A=\left(x_{1}, y_{1}\right)$ and moves to point $B=\left(x_{2}, y_{2}\right)$ with constant speed. Let the trajectory be parametrised by the variable $s$. The length of the trajectory, $\ell$, is a constraint on the problem as frequently one wishes to traverse the minefield in a prescribed time and/or distance.

The distance from any point $(x, y)$ on the trajectory to the $i$ th radar is 
denoted by $d_{i}$. The risk from each mine is proportional to $1 / d_{i}^{2}$ so the total risk travelling the trajectory is

$$
R=\int_{A}^{B} \sum_{i=1}^{N} \frac{\sigma_{i}}{d_{i}^{2}} d s,
$$

where $\sigma_{i}$ is the risk proportionality constant for each mine thus allowing for mines with differing strengths. The risk minimisation subject to the length and end point constraints can be formulated as a calculus of variations problem the details of which are given by Zabarankin et al. [10, Appendix]. The resulting equations are

$$
\begin{aligned}
& L_{x}-\frac{d}{d s}(\dot{x} L)=\lambda \ddot{x}, \\
& L_{y}-\frac{d}{d s}(\dot{y} L)=\lambda \ddot{y},
\end{aligned}
$$

where over-dots are $s$-derivatives, the risk functional is defined as

$$
L=\sum_{i=1}^{N} \frac{\sigma_{i}}{d_{i}^{2}},
$$

and $\lambda$ is a constant of integration that needs to be found. Rearranging equations (2a) and (2b) and substituting

$$
z_{1}=x, \quad z_{2}=\dot{x}, \quad z_{3}=y, \quad z_{4}=\dot{y},
$$

gives a system of four first order equations

$$
\begin{aligned}
& \dot{z}_{1}=z_{2}, \\
& \dot{z}_{2}=\frac{1}{\lambda+L}\left(L_{z_{1}}-z_{2} \frac{d L}{d s}\right), \\
& \dot{z_{3}}=z_{4}, \\
& \dot{z}_{4}=\frac{1}{\lambda+L}\left(L_{z_{3}}-z_{4} \frac{d L}{d s}\right) .
\end{aligned}
$$




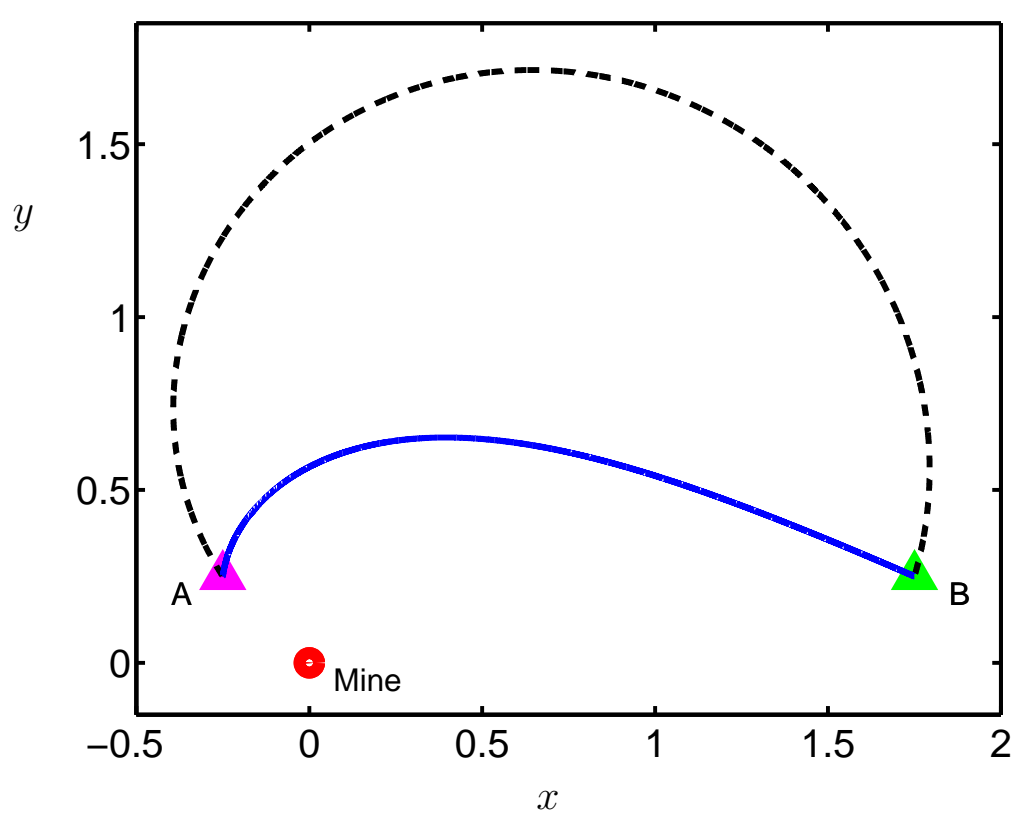

Figure 1: Two optimal routes for the one mine case. Solid line is for $\ell=2.5$ and dashed line is for $\ell=4.2$. The risk in traversing the routes are $R=4.1283$ and $R=3.2208$ respectively.

Since $s$ is an arc length there is also the additional constraint that

$$
\dot{z}_{1}^{2}+\dot{z}_{3}^{2}=1 \text {. }
$$

These equations need to be solved for the four variables $z_{1}, z_{2}, z_{3}, z_{4}$ and the integration constant $\lambda$ subject to the end points $\left(z_{1}(0), z_{3}(0)\right)$ and $\left(z_{1}(\ell), z_{3}(\ell)\right)$ being known.

\subsection{Numerical solution}

The system of equations is solved using the MATLAB ${ }^{\text {TM }}$ [4] routine BVP4C and independently checked using Fortran and the NAG [6] routine D02HBF. 


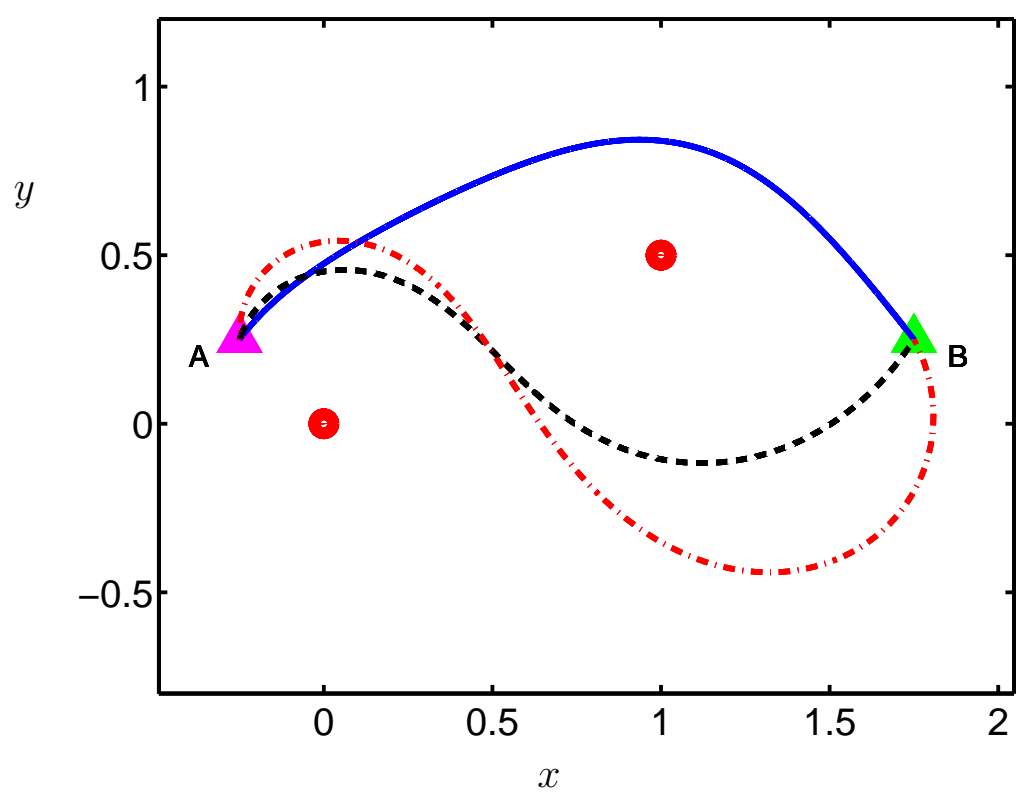

Figure 2: Two mine example showing three different optimal routes.

In general between 400 and 2000 points along the trajectory were needed for accurate results. Figure 1 shows trajectories for two different path lengths $(\ell=2.5,4.2)$ when the starting point is taken as $(-0.25,0.25)$ the end point $(1.75,0.25)$ and there is a single mine located at $(0,0)$. This configuration was chosen as it is the same configuration used for a related radar problem using a discrete method by Zabarankin et al. [10] and is therefore a check on the numerical method. The risk for each of the paths shown is $R=4.1283$ and $R=3.2208$ respectively. The optimal path in each case is, as expected, to be as far from the mine as possible while still satisfying the length constraint.

It is possible to find solutions for multiple mines. Figure 2 is an example of three optimal routes when there are two mines located at $(0,0)$ and $(1.0,0.5)$. There is now the possibility of having "inner" trajectories (going between the mines) and "outer" trajectories. In this example the "inner" route with a length $\ell=2.52$ has a lower risk $(R=11.0861)$ than an "outer" route of 


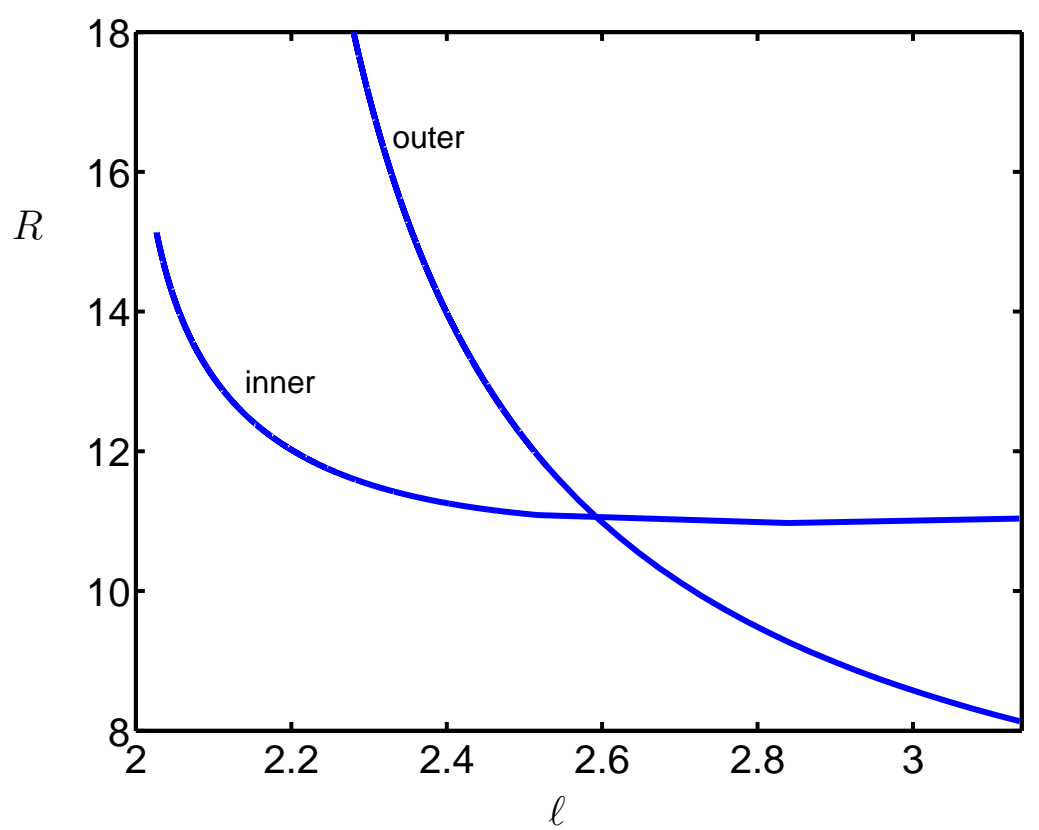

FiguRE 3: Risk versus path length for the two mine example showing the change over from "inner" to "outer" trajectory at approximately $\ell=2.6$.

the same length $(R=11.7214)$. There is now a distinct change in the route choice as the path length is increased. For short paths the "inner" route is the optimal choice but as the path length is increased and the risk reduced at some point the "outer" route becomes the preferred choice. This can be seen in Figure 3 which is a plot of the risk versus the path length for this two mine example. As the path length passes through approximately $\ell=2.6$ it is a lower risk to switch from the "inner" trajectory to the "outer" trajectory.

The solution method was found to be very sensitive to the initial trajectory and the location of the mines. To overcome the sensitivity to initial conditions it was necessary to initially have the mines at a great distance from the start and finish points and to iterate the process as the mines were slowly moved to their actual position. At each step of the iteration pro- 
cess we used the previous optimal trajectory as the new initial trajectory. This method has very slow convergence to the optimal solution and does not handle the sudden introduction of new mines well. These factors combine to make this method to be unsuitable for real time application of finding the optimal route through a minefield. An alternative method is therefore needed for onboard mine avoidance systems.

\section{Springs and masses approach}

\subsection{Formulation}

Due to the computational difficulties in obtaining solutions to the calculus of variations approach discussed in the last section we consider an approximate method that is faster and more robust. This method consists of distributing masses along the path and then evolving the position of these masses (and hence the trajectory) to minimise the risk. This work is based upon that of Bortoff [1] who studied the minimisation of risk of detection of an unmanned air vehicle traversing a region under enemy radar surveillance. The mines are assumed to repel the masses and there are springs connecting the masses that act to minimise the path length. This is shown schematically in Figure 4. By determining the forces acting on the masses a minimum energy solution can be found that is a balance between the mine repulsion and spring contraction.

Let there be $M$ masses distributed along the path at the points $\left(\bar{x}_{j}, \bar{y}_{j}\right)$ for $j=1, \ldots, M$ of mass $m_{j}$. The objective is then to find the position of these masses. Assume that the mines repel the masses with the repulsion proportional to the reciprocal of the square of the distance from a mine to a mass. The repulsion force acting on mass $j$ from all the mines is

$$
(\mathrm{RF})_{j}=\sum_{i=1}^{N} \frac{Q_{i}}{\left(d_{j i}\right)^{2}} \mathbf{n}_{j i},
$$




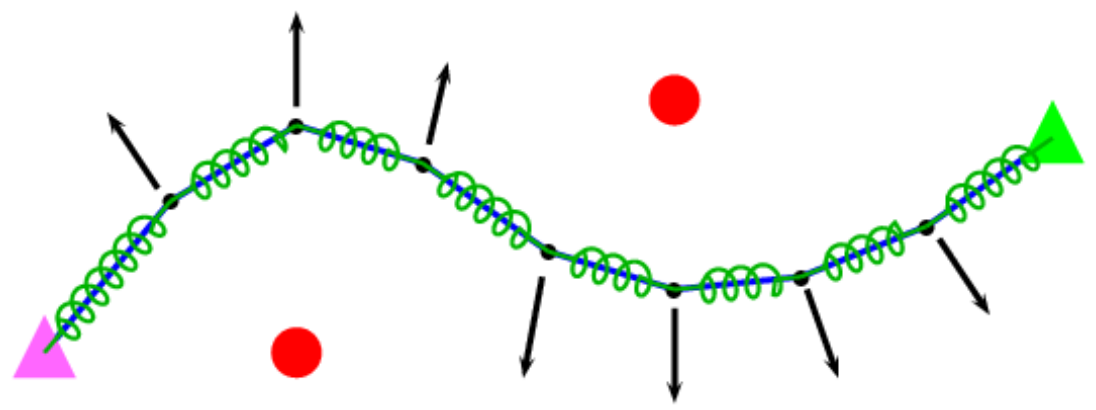

Figure 4: Schematic diagram showing the mines repelling the masses and the springs reducing the distance between the masses.

where $Q_{i}$ is the strength of mine $i$ and $\mathbf{n}_{j i}$ is the normal direction from mine $i$ to mass $j$. For brevity consider only the $x$-component of the forces (similar equations will hold for the $y$-component). The $x$-component of the repulsive force is then

$$
\left(\mathrm{RF}_{x}\right)_{j}=\sum_{i=1}^{N} \frac{Q_{i}}{\left(d_{j i}\right)^{3}}\left(\bar{x}_{j}-a_{i}\right),
$$

where $a_{i}$ is the $x$-component of the position of mine $i$. The $x$-component of the attractive forces acting on mass $j$ is due to its neighbours and is

$$
\left(\mathrm{AF}_{x}\right)_{j}=k\left(\bar{x}_{j+1}-\bar{x}_{j}\right)-k\left(\bar{x}_{j}-\bar{x}_{j-1}\right),
$$

where $k$ is the spring constant for the springs. There is also a spring damping force with an $x$-component

$$
\left(\mathrm{DF}_{x}\right)_{j}=b\left(\dot{\bar{x}}_{j+1}-\dot{\bar{x}}_{j}\right)-b\left(\dot{\bar{x}}_{j}-\dot{\bar{x}}_{j-1}\right),
$$

where $b$ is the damping coefficient for the springs.

Combining all the forces acting on each mass we obtain an ODE for the movement of the $x$-component of mass $j$ as

$$
m_{j} \ddot{\bar{x}}_{j}=\sum_{i=1}^{N} \frac{Q\left(\bar{x}_{j}-a_{i}\right)}{d_{j i}^{3}}+k\left(\bar{x}_{j+1}-\bar{x}_{j}\right)-k\left(\bar{x}_{j}-\bar{x}_{j-1}\right)+
$$




$$
+b\left(\dot{\bar{x}}_{j+1}-\dot{\bar{x}}_{j}\right)-b\left(\dot{\bar{x}}_{j}-\dot{\bar{x}}_{j-1}\right) \quad \text { for } j=1, \ldots, M \text {. }
$$

Similarly we derive an ODE for the $y$ coordinate $m_{j} \ddot{\bar{y}}_{j}$. Together these form a system of $4 M$ first order ODEs that govern the movement of the masses toward an optimum trajectory.

\subsection{Numerical solution}

From an initial mass distribution along a path integrate the system of ODEs forward in time until a local minimum energy state (steady state) is obtained. This then represents the balance between the mine repulsive and spring attractive forces. By adjusting the repulsion coefficients, $Q_{i}$, and the spring constants, $k$, one determines an optimal route for a given path length. Since this method only requires the integration of a system of initial value ODEs forward in time to a steady state, it is fast and robust. Even for large numbers of masses, which are necessary to obtain a smooth continuous trajectory, this method is suitable for real time calculations.

Figure 5 shows an example of the optimal (minimum risk) route through a large minefield where in this example the mines are all taken to have the same repulsion strength, $Q_{i}=Q$ for $i=1, \ldots, N$. The optimal route is found by adjusting the ratio of the mine repulsion to the spring constant until a minimal risk route is found. In this case, minimal risk occurs when $Q / k=0.8$ for a path length of $\ell=2.0931$ and a risk of $R=131.89$ using $M=50$ masses along the route.

Figure 5 hints at a potential problem with this method. As a result of the mines repelling the masses they are distributed to areas of low risk and away from areas of high risk such as when the route has been forced by the length constraint to pass near a mine. This tendency reduces the accuracy of the method. It can be overcome by increasing the number of masses used or by distributing the masses so that areas of high risk have a higher density of 


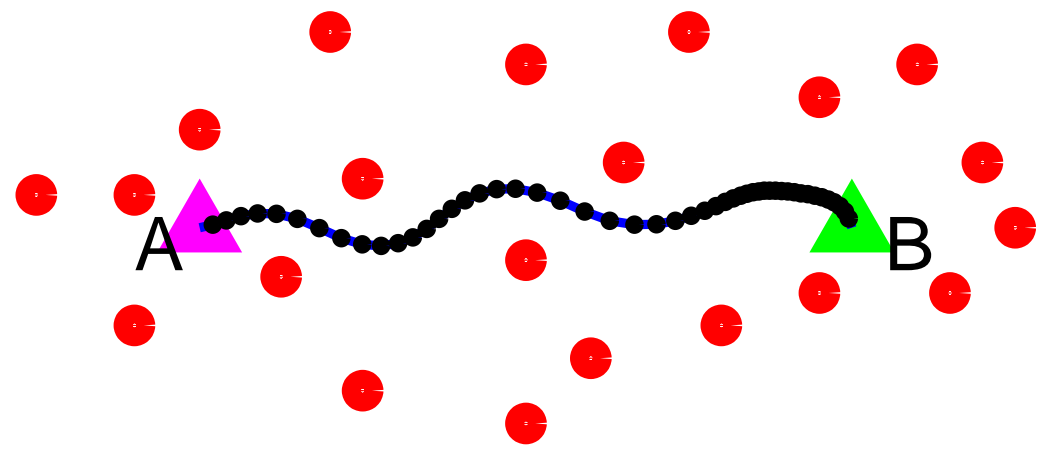

Figure 5: An example of the optimal route through a minefield with multiple mines present using the springs and masses method.

masses. The latter of these two is preferred if computational speed is desired for real time application.

\section{Conclusion}

Two methods have been presented for the calculation of a minimal risk path through a minefield in a continuous setting. These methods differ from the classic discrete formulations of these problems in that the discretisation is along the trajectory and not over the whole region. In general this makes these continuous methods more efficient than the classic whole of region discretisation methods.

The convergence of the solutions obtained via the calculus of variations method presented here was found to be very slow and so not suitable for real time implementation. In contrast, the springs and masses approach is robust, fast and efficient. However, the solution obtained using the springs and masses method is only an approximation to the actual problem. 
In the springs and masses method the path length is an outcome not an input; in contrast in the calculus of variations method it is one of the inputs. To obtain a given path length using the springs and masses method the ratio of the mine repulsion to the spring constant must be iterated upon. Because this method is so much faster than the calculus of variations method this is not an impediment.

In real life scenarios the locations of all mines are not necessarily known. Often there are what are referred to as "pop up" threats [1]. These are mines that are only discovered once the vehicle has begun traversing the route through the minefield. The springs and masses method is able to handle these "pop up" threats much more easily than the calculus of variations method since the springs and masses method is a "local" method in that each mass is affected more by mines near it rather than those far away. Due to the "local" adaptability of the springs and masses method the route can be varied in real time.

\section{References}

[1] S. A. Bortoff. Path planning for UAVs. In Proceedings of the American Control Conference, pages 364-368, Chicago, Illinois, June 2000. http://www.ieeexplore.ieee.org/. C300, C304

[2] J. Krozel and D. Andrisani II. Navigation path planning for autonomous aircraft: Voronoi diagram approach. AIAA, J. of Guidance, Control and Dynamics, 13:1152-1154, 1990. http://www . aiaa. org/content. cfm? pageid=322\&lupubid=23. C295

[3] J. J. Leary. Search for a stealthy flight path through a hostile radar defense network. Master's thesis, Naval Postgraduate School, Monterey, CA, 1995. http://stinet.dtic.mil/. C295 
[4] MATLAB ${ }^{\mathrm{TM}}$. The Mathworks, Inc. http://www. mathworks.com. C297

[5] R. Murphey, S. Uryasev, and M. Zabarankin. Trajectory optimization in a threat environment. Technical Report 9, University of Florida, July 2003. C294, C295

[6] NAG. Numerical algorithm group. http://www.nag.co.uk. C297

[7] M. C. Novy. Air vehicle optimal trajectories for minimization of radar exposure. Master's thesis, Air Force Institute of Technology, Wright-Patterson Air Force Base, Ohio, 2001. http://stinet.dtic.mil/. C295

[8] L. C. Thomas and J. N. Eagle. Criteria and approximate methods for path-constrained moving-target search problems. Naval Research Logistics, 42:27-38, 1995. C295

[9] J. L. Vian and J. R. More. Trajectory optimization with risk minimization for military aircraft. AIAA, J. of Guidance, Control and Dynamics, 12(3):311-317, 1989.

http://www. aiaa.org/content. cfm?pageid=322\&lupubid=23. C294

[10] M. Zabarankin, S. Uryasev, and P. Pardalos. Optimal risk path algorithms. In R. Murphey and P. Pardalos, editors, Cooperative control and optimization, volume 66, pages 271-30. Kluwer Academic Publishers, Dordrecht, 2002. C295, C296, C298 


\section{Author addresses}

1. G. N. Mercer, School of Physical, Environmental and Mathematical Sciences, University of New South Wales at the Defence Force Academy, Australia.

mailto:g.mercer@adfa.edu.au

2. H. S. Sidhu, School of Physical, Environmental and Mathematical Sciences, University of New South Wales at the Defence Force Academy, Australia. 